\title{
Step-Tracking Movements of the Wrist in Humans. I. Kinematic Analysis
}

\author{
Donna S. Hoffman and Peter L. Strick \\ Research Service, V.A. Medical Center, Departments of Neurosurgery and Physiology, SUNY Health Science \\ Center at Syracuse, Syracuse, New York 13210
}

We have examined the kinematics of the initial trajectory of step-tracking movements performed by human subjects. Each subject tracked a target that required $5-30^{\circ}$ of radial or ulnar deviation of the wrist. All movements were to be performed as accurately as possible. Speed instructions were given before each trial.

When subjects performed different amplitude movements following the same speed instruction, the peaks of velocity, acceleration, and jerk were linearly related to peak displacement. The peaks of velocity, acceleration, and jerk also changed when the speed instruction was altered. Thus, for any given movement, the peak values of the derivatives of displacement were dependent on both movement amplitude and intended speed. As a result, the peak values of the derivatives cannot be used by themselves to control or monitor peak displacement.

When subjects performed different amplitude movements following the same speed instruction, movement duration tended to remain constant. In contrast, movement duration changed when the speed instruction was altered. Movements performed when subjects intended to move slowly had longer durations than when subjects intended to move quickly. These results suggest that subjects volitionally alter intended speed by selecting different movement durations.

When both movement amplitude and intended speed were varied, the peak displacement of a step-tracking movement was linearly related to the product of 2 kinematic variables: (1) the initial peak of a derivative of displacement (either velocity, acceleration, or jerk) and (2) movement duration. On the basis of our observations, we propose that central commands generate step-tracking movements of different amplitudes and intended speeds by adjusting both the magnitude and duration of a derivative of displacement.

Movements of a single joint are associated with complicated patterns of muscle activity (e.g., Wachholder, 1928). Thus, even when generating these movements, the nervous system is faced with a potentially formidable task. Recently, there have been several attempts to define movement parameters that, if controlled or monitored by the nervous system, would simplify movement generation (see, for a recent review and commentaries, Stein, 1982). Most of these studies have focused on the control of relatively slow limb movements (e.g., Bizzi ct al., 1984; Lestienne et al., 1981; Polit and Bizzi, 1979). We have attempted to identify the variables controlled by the CNS when

Received Oct. 3, 1985; revised May 2, 1986; accepted May 6, 1986.

This work has been supported in part by funds from the Veterans Administration Medical Research Service, USPHS Grant 02957, and the Department of Neurosurgery. The authors thank Ms. Laura Westfall for her expert technical assistance.

Correspondence should be addressed to Dr. Peter L. Strick, Research Service (151), V.A. Medical Center, Syracuse, NY 13210.

0270-6474/86/113309-10\$02.00/0 subjects perform more rapid wrist movements in a step-tracking task. One of our reasons for studying step-tracking movements was that the pattern of muscle activity associated with them is well defined (e.g., Angel, 1974; Hallett and Marsden, 1979; Hallett et al., 1975; Wachholder and Altenburger, 1926; Waters and Strick, 1981). During this type of movement, distinct bursts of activity are observed in agonist and antagonist muscles. We sought to determine whether the initial bursts of muscle activity were associated with particular kinematic parameters.

The first step in this analysis was to define the kinematic parameters associated with step-tracking movements. Most prior studies have focused only on movements performed following a constant speed instruction. These studies observed that changes in limb displacement were associated with proportional changes in limb velocity (e.g., Taylor and Birmingham, 1948). On the basis of this result, Freund and Budingen (1978) proposed the "speed control" hypothesis, which states that movements of different amplitudes are generated by adjusting a single kinematic variable, limb velocity.

An important feature of step-tracking movements that has not received sufficient consideration is that subjects can intentionally vary peak velocity when making movements of a single amplitude (see, however, Brown and Cooke, 1981; Lestienne, 1979; and Marsden et al., 1983). Therefore, we have examined the characteristics of step-tracking movements performed when both target displacement and "intended speed" were varied. The results of our analysis suggest that the nervous system specifies the initial trajectory of a step-tracking movement by controlling 2 kinematic variables: (1) the peak magnitude of a derivative of displacement and (2) the duration of a derivative of displacement. In a subsequent paper, we will present evidence that separate bursts of muscle activity control each of the 2 kinematic variables (D. S. Hoffman and P. L. Strick, unpublished observations). Preliminary reports of some of these findings have been presented (Hoffman and Strick, 1982, 1984).

\section{Materials and Methods}

\section{Experimental setup}

Five human subjects (aged 25-38) without any history of neurological abnormalities sat in a chair; the forearm and elbow of their dominant limb supported. The subjects grasped the handle of a manipulandum (Fig. 1) that was mounted on a sturdy support in front of them. The manipulandum handle rotated freely about the horizontal and vertical axes. Each subject's forearm was positioncd carcfully so that the centers of rotation of the manipulandum and the wrist coincided. Two potentiometers were coupled to the device and measured the angle of the wrist in the planes of flexion-extension and radial-ulnar deviation. The manipulandum was a lightweight, low-friction device which, we believe, minimally alters wrist movements.

Each subject sat approximately $70 \mathrm{~cm}$ in front of a large-screen oscilloscope that displayed a "cursor" and a "target." The cursor was a small spot of light (approximately $5 \times 5 \mathrm{~mm}$ ) that moved in proportion to the subject's wrist movements. One degree of wrist movement moved 


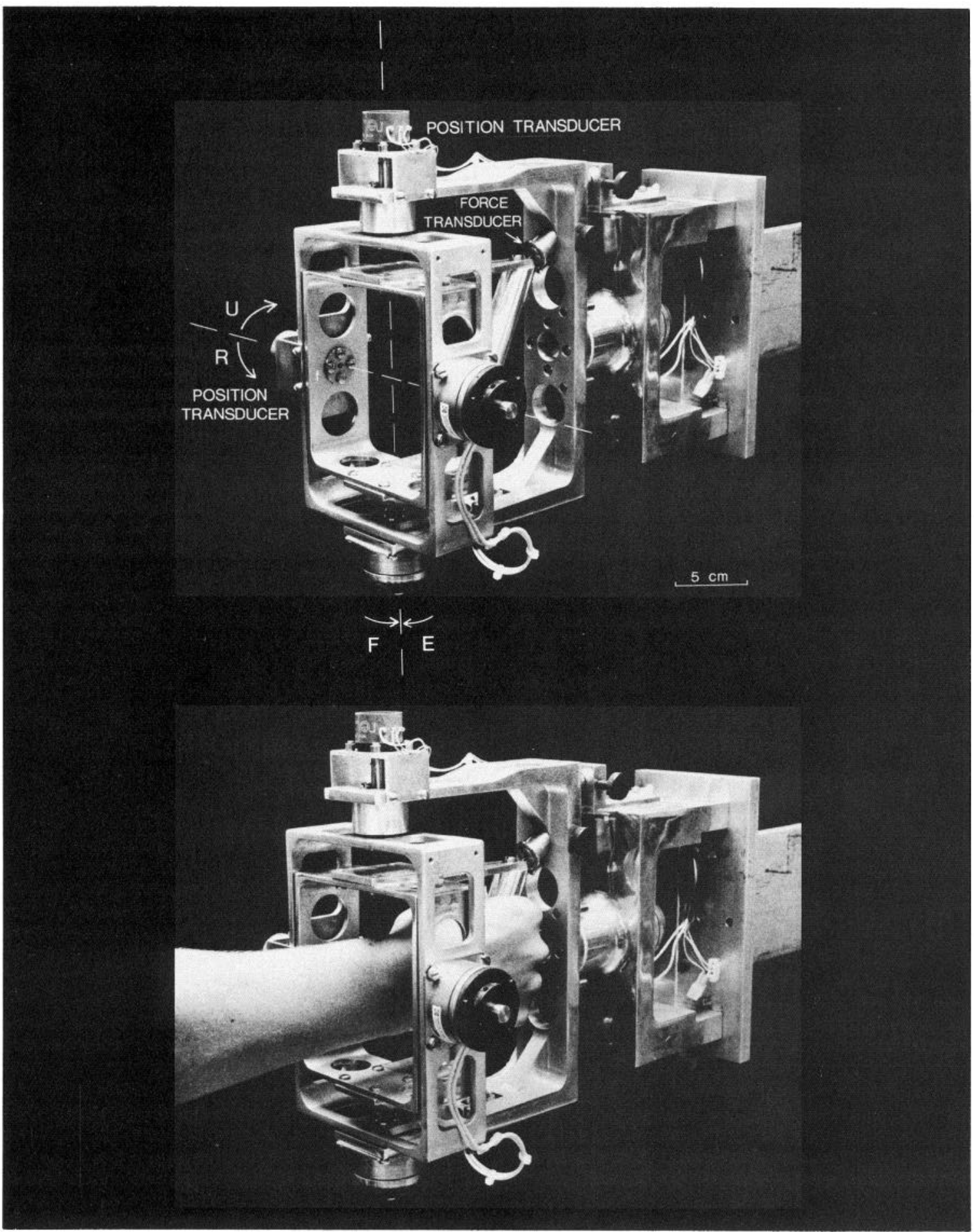

Figure 1. Two-axis manipulandum. Top, Two position transducers monitor the 2 axes of wrist movement. The transducer at the left monitors radial $(R)$-ulnar $(U)$ deviation. The transducer on the top monitors flexion $(F)$-extension $(E)$. The 2-axis force transducer was not employed in these experiments. Bottom, The position of the forearm in the manipulandum. The handle of the manipulandum was adjusted toward or away from the subject, until the centers of rotation of the manipulandum and the wrist joint matched. The wrist is shown in the neutral position.

the cursor approximately $4.5 \mathrm{~mm}$ on the screen. For a right-handed subject, the cursor moved left for flexion movements, right for extension movements, up for radial deviations, and down for ulnar deviations of the wrist. The target was displayed as an open square whose inside diameter equaled $2.5^{\circ}$ of wrist movement. It indicated where the subject should place the cursor. The location of the target on the screen was determined by a computer (DEC PDP 11/34) that was programmed to alter target location according to task requirements.

\section{Task}

Each subject was asked to perform a step-tracking task that required radial or ulnar deviations of the wrist. In order to perform these movements, the subject had to prevent flexion or extension, since the handle could move easily in all directions. When movements were made in the radial direction, the initial position of the target required $10^{\circ}$ of ulnar deviation. When movements were made in the ulnar direction, 


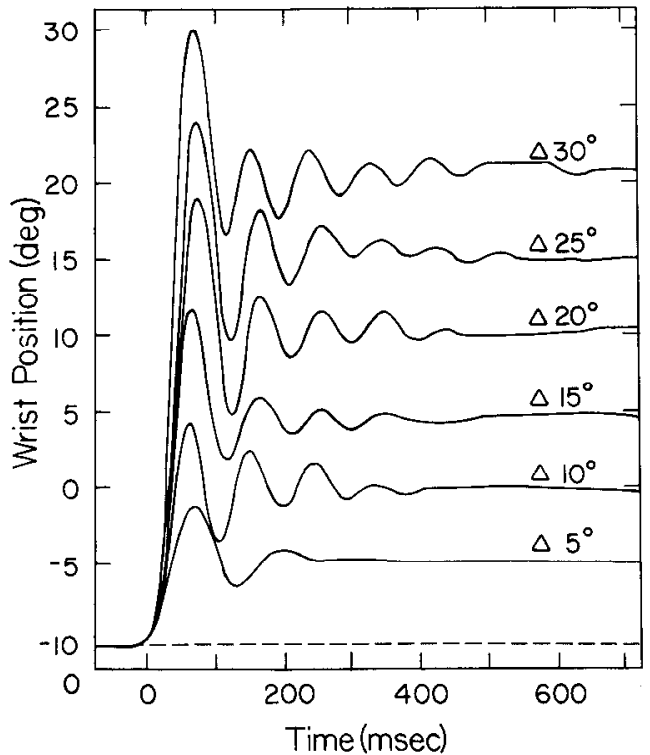

Figure 2. Single trials of step-tracking movements made by 1 subject. Subjects were instructed to move "as fast and as accurately as possible" to different targets. Targets required $5^{\circ}-30^{\circ}$ wrist movements in the radial direction, starting from an initial position of $10^{\circ}$ ulnar deviation. Actual wrist angle is indicated by the scale on the left (negative numbers, ulnar deviation; positive numbers, radial deviation; 0 , neutral position, illustrated in Fig. 1).

the initial position of the target required $20^{\circ}$ of radial deviation. To initiate a trial, the subject centered the cursor in the target and maintained this position for a variable "hold" period (1-2 sec). The target then jumped to a new location. The subject, when ready, was required to move in a single step, as accurately as possible, to this new position.
After another variable hold period, the target was moved back to the initial position. The subject was permitted to return the cursor to this location in any manner desired. Since the experimental paradigms described below required large numbers of trials, subjects were given frequent rest periods and were observed for possible signs of fatigue.

In the first series of trials for each experimental session, the subject was instructed to perform movements to the new target position "as fast as possible." Target jumps required between $5^{\circ}$ and $30^{\circ}$ changes in wrist angle. The same target location was presented for a block of 50 trials. The order in which the different target locations were presented was randomized.

Four of the 5 subjects were asked to perform a second series of trials. In these trials, the subject was given 3 different speed instructions: to "move as fast as possible"; "move at your natural speed"; and "move at half your natural speed." We will use the term "intended speed" to indicate the subject's interpretation of these instructions. Target jumps required $10^{\circ}$ and $20^{\circ}$ changes in wrist angle. The same displacement and speed instructions were presented for a block of 30-50 trials.

One subject was reexamined in a separate experimental session using a different protocol. The subject was asked to perform about 30 movements to a target "as fast as possible." Then, the subject was asked to move to the same target while the speed instruction was randomly varied. Instructions were to "move a little slower," "move a little faster," or "move at the same speed." Target jumps required $5^{\circ}, 15^{\circ}$, and $25^{\circ}$ changes in wrist angle. The same displacement was presented for a block of 150 trials.

\section{Data acquisition and analysis}

While the subject performed the step-tracking movements, signals from the potentiometers and an analog code representing various task-related events were monitored on a storage oscilloscope. These signals were recorded on an FM tape recorder (HP 8868A) for later analysis. The computer was used off-line to digitize the data from individual trials at $2.5 \mathrm{kHz}$. The digitized potentiometer signals were low-pass filtered numerically by the computer (cutoff frequency $=200 \mathrm{~Hz}$ ). Filtering was applied in the backward, as well as the forward, direction in order to correct for phase shifts. Filtered signals were then successively differentiated to derive velocity, acceleration, and jerk (change in acceleration)
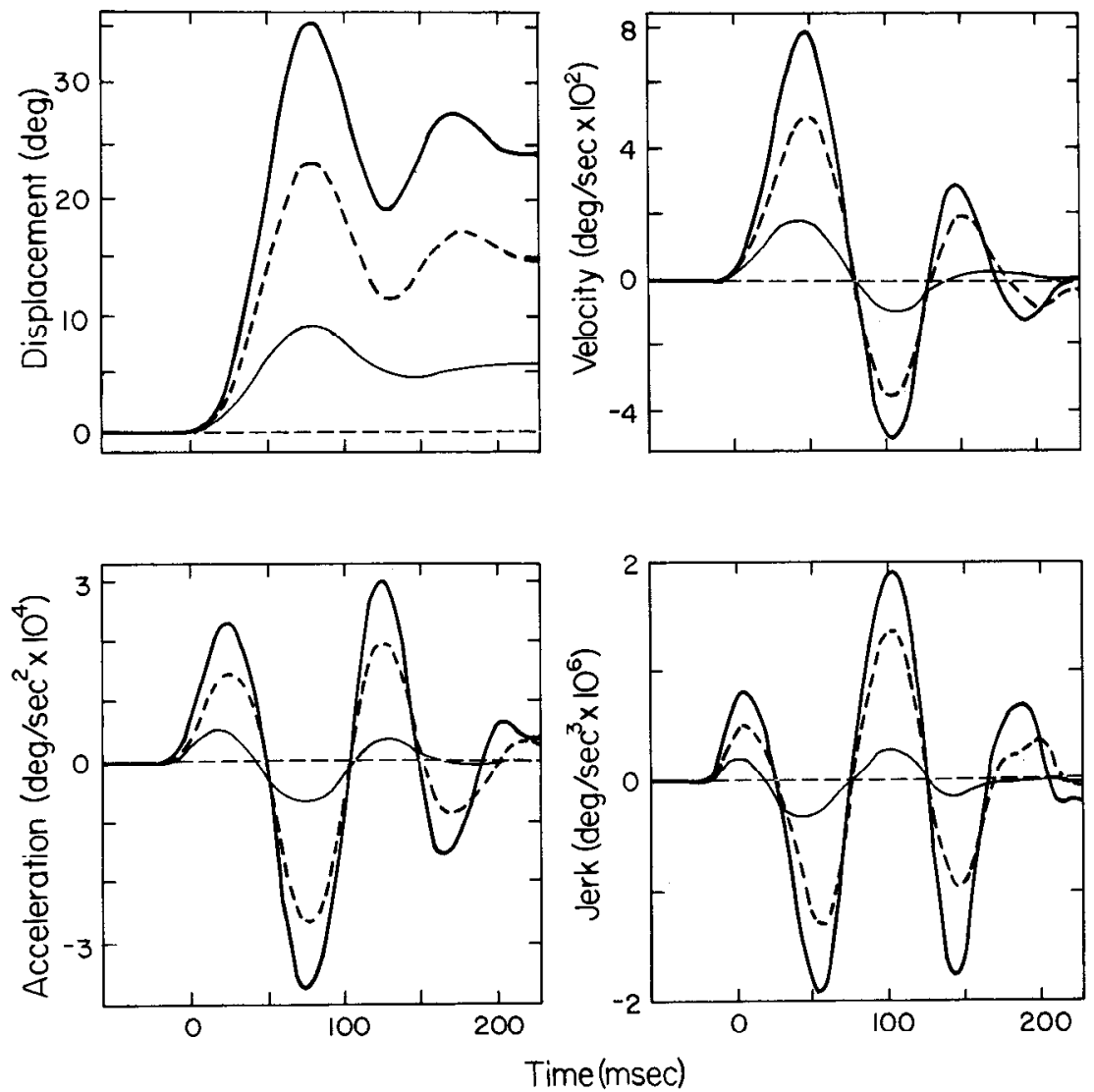

Figure 3. Averages of step-tracking movements performed as fast as possible. Thick solid line, average of 40 movements to the $25^{\circ}$ target; dashed line, average of 42 movements to the $15^{\circ}$ target; thin solid line, average of 27 movements to the $5^{\circ}$ target. Movements were performed in the radial direction starting from an initial position of $10^{\circ}$ ulnar deviation. All trials are from the same subject. Note that velocity, acceleration, and jerk each have 1 positive peak during the initial trajectory (i.e., the period from movement onset to the first peak in displacement). Also, the magnitudes of these peaks grade with changes in movement amplitude. 
Figure 4. Relationship between peak displacement and peak velocity for movements to different targets performed "as fast as possible." $A$, Individual trials and linear-regression line of 1 subject performing movements in the radial direction. The averages of these trials were shown in Figure 3. B, Linear-regression lines of 5 subjects. For each line, $r>0.98$.
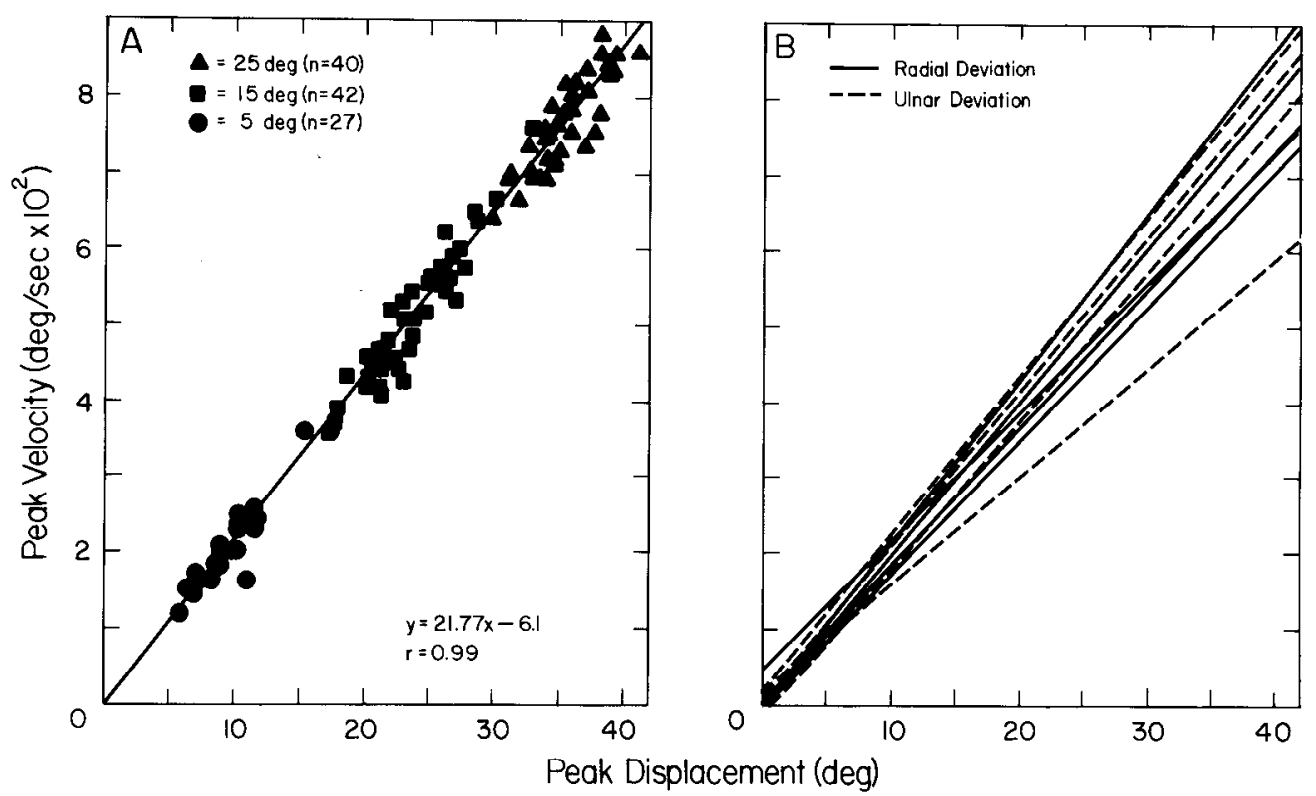

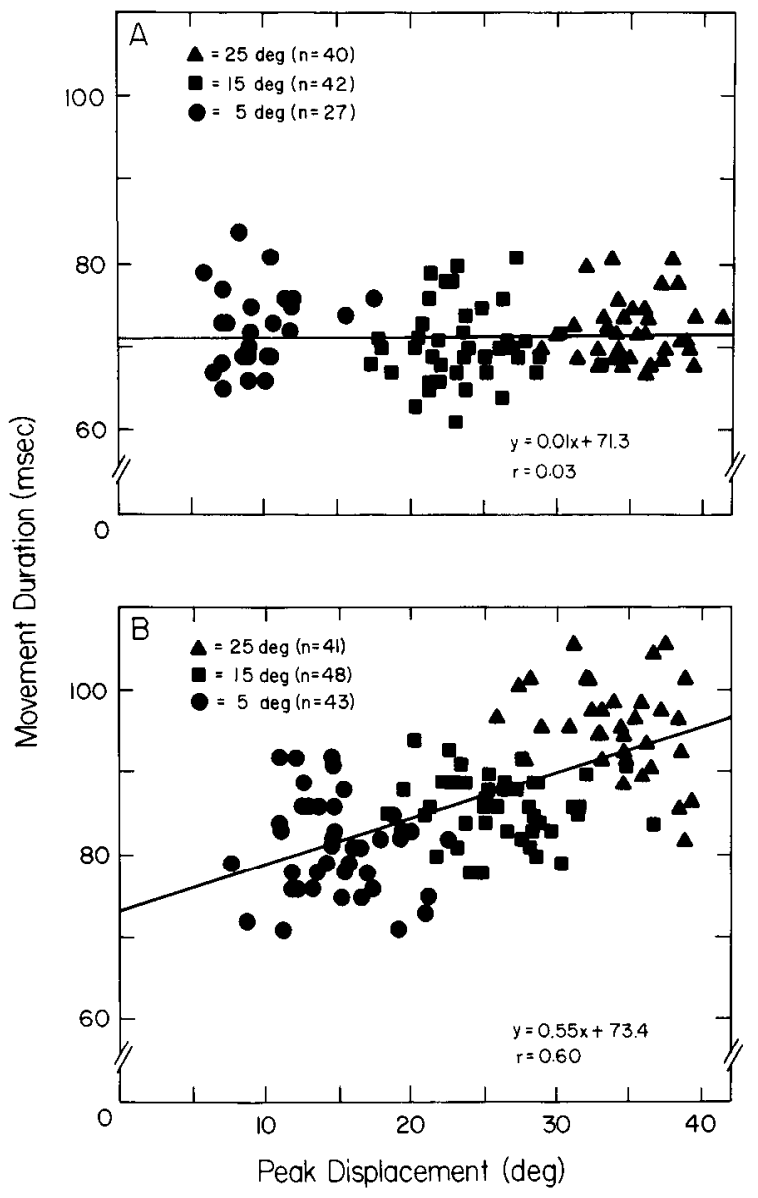

Figure 5. Relationship between peak displacement and movement duration for movements to different targets performed "as fast as possible." Movement duration was defined as the time from movement onset to the first peak in displacement (i.e., the time between movement onset and the next 0 value of velocity). $A$, Individual trials and linearregression line of 1 subject. The averages of these trials were shown in Figure 3. The slope of this linear-regression line is not statistically significant $(p>0.05)$. $B$, Individual trials and linear-regression line of another subject. The slope of this linear regression line is statistically significant $(p<0.01)$.
(Wood, 1982). The initial trajectory of each movement was analyzed for the following variables: movement duration and peaks of displacement, velocity, acceleration, and jerk. Movement duration was defined as the time from movement onset to the first peak in displacement (i.e., the time between movement onset and the next 0 value of velocity). Unless otherwise noted, only correlations with $p<0.01$ were considered to be significant.

\section{Results}

Movements performed "as fast as possible"

Our analysis has focused on the initial trajectory of step-tracking movements, i.e., that part of the movement from its onset 10 the first peak in displacement. This period was characterized by a rapid, but smooth, change in position that generally overshot the target (Figs. 2, 3). The overshoot was greatest for the largest displacements and was not eliminated by practice. The overshoot was followed by several stereotyped oscillations in displacement. The frequency of the oscillations varied among different subjects, ranging from 8 to $11 \mathrm{~Hz}$ for movements made to $25^{\circ}$ targets.

As part of our analysis, we examined the first, second, and third derivatives of displacement (velocity, acceleration, and jerk). Each of these derivatives changed smoothly and had only 1 positive peak during the initial trajectory (Fig. 3). Thus, the initial movement trajectory was performed in a single step, rather than in multiple, smaller steps.

The magnitudes of the initial peaks of velocity, acceleration, and jerk varied directly with the amplitude of the initial trajectory (Fig. 3). The best relationship between peak displacement and the peaks of the derivatives is shown for 1 subject in Figure $4 A$. There was a striking correlation between peak displacement and peak velocity for these trials $(r=0.99)$. The linear-regression line in this graph has a steep positive slope $(21.77 \mathrm{deg}$ $\mathrm{sec}^{-1} \mathrm{deg}^{-1}$ ) and a $y$-intercept that is not significantly different from zero. The 4 other subjects we examined displayed similar strong correlations between peak displacement and peak velocity $(r>0.98)$. Smaller, but highly significant, correlations also were observed between peak displacement and the peaks of acceleration and jerk. For example, the correlations for the trials illustrated in Figure $4 A$ were $r=0.98$ (versus peak acceleration) and $r=0.95$ (versus peak jerk). These observations on steptracking movements performed "as fast as possible" suggest 2 conclusions. First, different amplitude movements are produced 

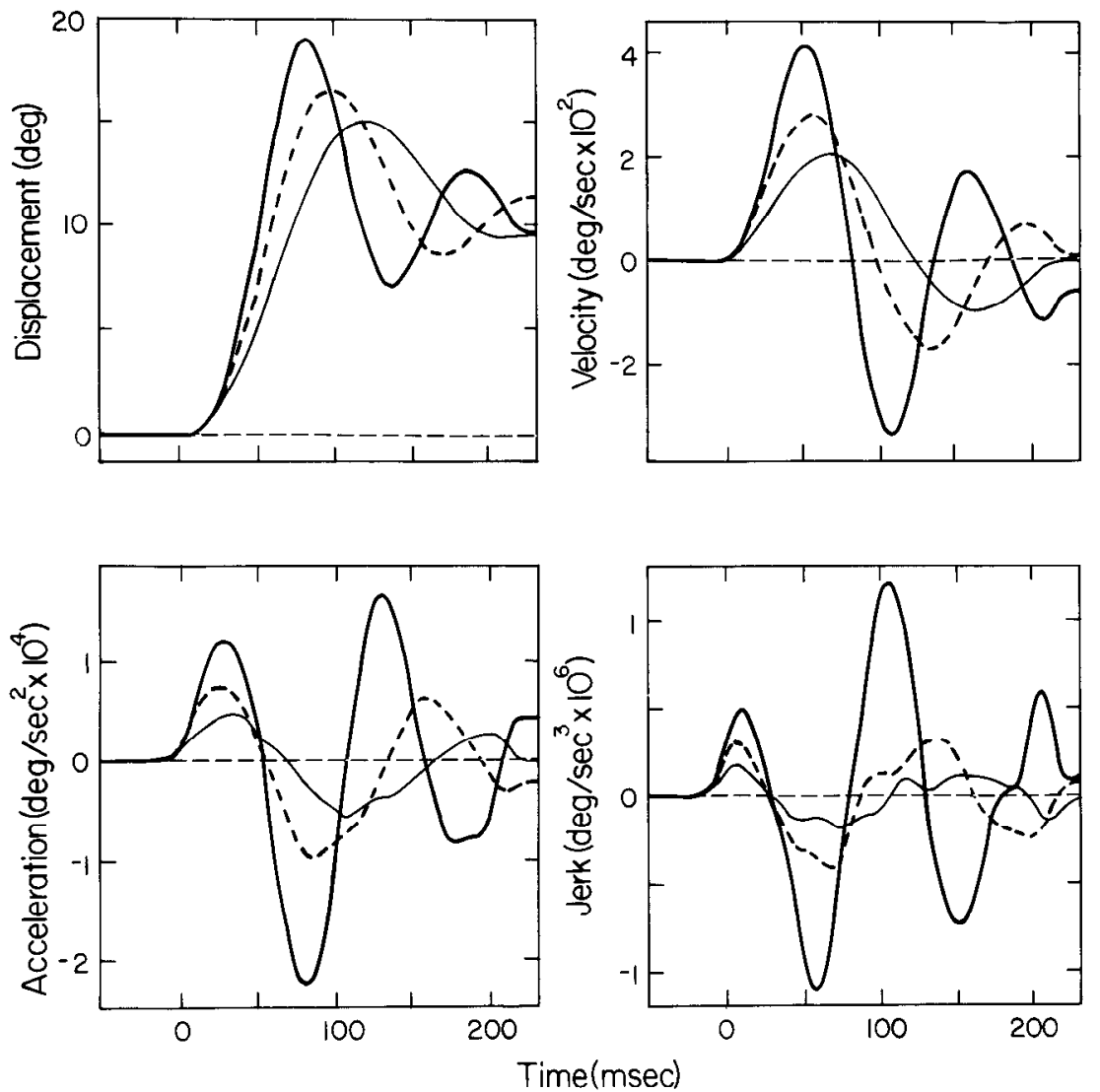

Figure 6. Averages of step-tracking movements performed at different intended speeds. This subject made movements to the $10^{\circ}$ target. Movements were performed in the radial direction, starting from an initial position of $10^{\circ}$ ulnar deviation. Thick solid line, average of 56 movements performed "as fast as possible"; dashed line, average of 42 movements performed at a "natural speed"; thin sol$i d$ line, average of 45 movements performed at "half natural speed."

by grading the first 3 derivatives of displacement. Second, derivative feedback during the initial trajectory could provide an early predictive measure of the subsequent peak displacement.

Four subjects were asked to perform step-tracking movements "as fast as possible" in the ulnar as well as the radial direction (Fig. $4 B$ ). The regression lines of the relations between peak displacement and peak velocity for the 2 directions of movement had similar average slopes (radial $=19.1 \mathrm{deg} \mathrm{sec}^{-1} \mathrm{deg}^{-1}$; ulnar $\left.=18.9 \mathrm{deg} \mathrm{sec}^{-1} \mathrm{deg}^{-1}\right)$. The movement durations for the 2 directions of movement were also similar (see below). One interpretation of these results is that, when mechanical conditions differ (such as for the 2 directions of movement), motor output is adjusted to maintain a constant relationship between peak displacement and peak velocity.

We also examined whether movement duration varied for different amplitude movements. We defined movement duration as the duration of the initial trajectory, which is the time from movement onset to the next zero-crossing of velocity (Fig. 3 ). For 2 of the subjects, movement duration and peak displacement were not correlated (Fig. $5 A$ ). Thus, despite our repeated request that subjects move "as fast as possible," the same amount of time was required to complete both small- and largeamplitude movements.

The 3 other subjects had small, but significant, correlations between movement duration and peak displacement $(r$ 's $=0.45$, 0.60 , and 0.73 ) (Fig. $5 B$ ). The linear-regression lines relating peak displacement to movement duration for these subjects had an average slope of $0.55 \mathrm{msec} / \mathrm{deg}$ for movements in the radial direction and $0.47 \mathrm{msec} / \mathrm{deg}$ for the ulnar direction. Thus, the duration of large movements $\left(25^{\circ}\right)$ was only $20 \%$ greater than that of small movements $\left(5^{\circ}\right)$. In contrast, peak velocity increased $200 \%$ on the same trials. We did not quantitatively examine the duration of acceleration or jerk. However, inspec- tion of the averages of these derivatives (e.g., Fig. 3) indicated that their duration also showed only small variations with changes in movement amplitude. Taken together, our observations suggest that different amplitude movements performed "as fast as possible" were produced primarily by grading the magnitude rather than the time course of the derivatives of displacement.

In 4 subjects, we cxamincd the variations in the magnitude and duration of the derivatives of displacement for repeated movements to a single target $\left(5^{\circ}, 15^{\circ}, 25^{\circ}\right)$. Peak velocity and peak displacement were always highly correlated for movements to 1 target $(r>0.87)$. In contrast, movement duration and peak displacement were not significantly correlated for the same movements $(r<0.23 ; p>0.05)$. These observations suggest that the variations in peak displacement for movements to a single target were also primarily due to variations in the magnitude, but not the duration, of the derivatives of displacement.

\section{Movements performed at different intended speeds to a single target}

Regardless of the speed instruction, limb displacement and its derivatives changed smoothly and had 1 positive peak during the initial trajectory (Fig. 6). Thus, step-tracking movements performed at slower intended speeds were like those made "as fast as possible." However, there were some alterations in steptracking movements when the speed instruction was changed. The most dramatic alteration occurred in the time course of the derivatives of displacement. Movement duration increased an average of $67 \%$ when the speed instruction was changed from move "as fast as possible" to move at "half natural speed." With different speed instructions, we observed initial trajectories as short as $58 \mathrm{msec}$ and as long as $218 \mathrm{msec}$. Thus, there was a striking contrast between the relatively fixed movement duration of subjects in moving to different targets "as fast as 
Figure 7. A, Relationship between peak displacement and peak velocity for movements performed at different intended speeds. All movements were from 1 subject to $10^{\circ}$ and $20^{\circ}$ targets. The symbols and bars indicate the mean $\pm \mathrm{SD}$. The equations for the linear regression lines are: for "fast," $y=17.74 x-6.35(r=0.98)$; for "natural," $y=14.92 x-16.47(r=$ 0.99 ); and for "half natural," $y=$ $10.10 x+16.72(r=0.90) . B$, Relationship between peak displacement and movement duration for the same trials as in $A$. The equations for the linear regression lines are: for "fast," $y=-0.16 x+88.3(r=-0.19 ; p>$ 0.05 ); for "natural," $y=-0.17 x+$ $119.4(r=-0.12 ; p>0.05)$; and for "half natural," $y=1.64 x+119.5(r=$ $0.33 ; p<0.01$ ).
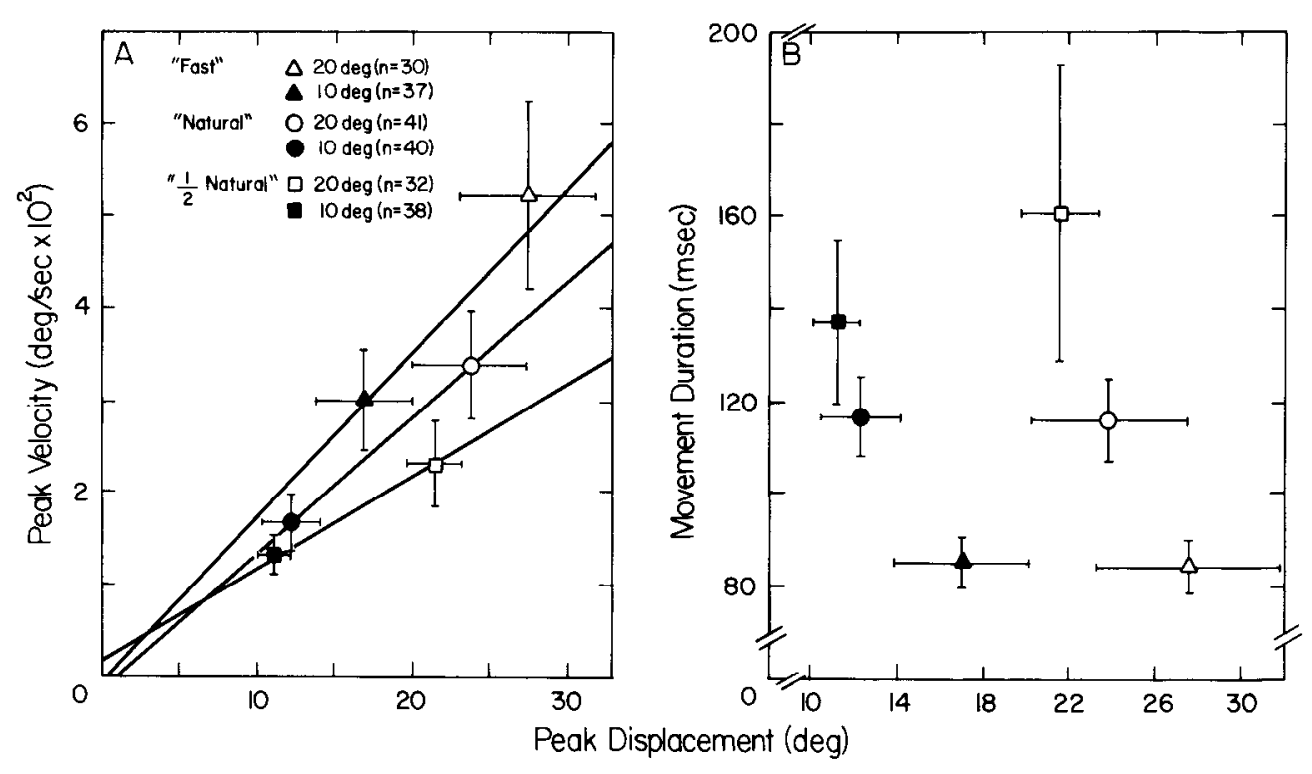

possible" (e.g., Fig. 3) and the variable movement duration of the same subjects in moving to 1 target at different intended speeds (e.g., Fig. 6).

Changes in the speed instruction also influenced the magnitude of the initial overshoot. Peak displacement decreased an average of $15 \%$ when the speed instruction was changed from move "as fast as possible" to move at "half natural speed." In fact, some movements performed at "half natural speed" undershot the target. Changes in intended speed from "as fast as possible" to a slower speed also reduced the frequency and number of terminal oscillations (not illustrated). Thus, movements performed at slower intended speeds were more damped than movements performed "as fast as possible."

Different speed instructions also led to alterations in derivative magnitude. For example, peak velocity decreased an average of $46 \%$ when the speed instruction was changed from move "as fast as possible" to move at "half natural speed." Peak jerk decreased an average of $59 \%$ on the same trials. These observations, together with those presented in the previous section, indicate that 2 factors influence the magnitude of the derivatives: (1) movement amplitude and (2) speed instruction.

\section{Movements of different amplitudes and intended speeds}

We examined whether movement duration varied when subjects made different amplitude movements following the "natural" and "half natural speed" instructions. Peak displacement and movement duration were not significantly correlated for the "natural speed" movements of 2 subjects (e.g., Fig. 7B). The "natural specd" movements of the other 2 subjects cxamined, and the "half natural speed" movements of all subjects $(n=3)$, had small but statistically significant correlations between peak displacement and movement duration $(r=0.33-0.51$; e.g., Fig. $7 B$ ). These results are similar to those observed when subjects performed movements "as fast as possible" (Fig. 5, $A, B$ ). Thus, when the speed instruction was kept constant, grading of movement duration in relation to movement amplitude was small and inconsistently observed.

When subjects performed different amplitude movements with a constant speed instruction (moving at either "natural" or "half natural" speed), the peaks of velocity, acceleration, and jerk varied directly with peak displacement (e.g., Fig. 7A). For movements following either speed instruction, the correlations between peak displacement and peak velocity were high for all subjects $(r=0.84-0.99)$. Smaller, but highly significant, correlations were observed between peak displacement and the peaks of the other derivatives $(r=0.64-0.91)$. These findings are similar to those observed when movements were performed "as fast as possible" (Fig. 4A). Thus, our observations on movements with a constant speed instruction indicate that different amplitude movements were produced primarily by grading the magnitude rather than the time course of the derivatives of displacement.

The slopes of the regression lines for the relationships between peak displacement and the peaks of the derivatives were dependent on the speed instruction (Fig. 7A). The shallowest slopes were associated with the "half natural speed" movements; the steepest slopes were observed with the "as fast as possible" movements; and the slopes for "natural speed" movements were intermediate. These results indicate that the relationships between displacement and its derivatives were not fixed, but were determined by the speed instruction.

The observation that speed instructions influence the relationship between displacement and its derivatives has an important consequence. It shows that movement amplitude is not controlled solely by grading the peaks of velocity, acceleration, and jerk when movements are made at different intended speeds. Thus, movements with different peak velocities may have the same peak displacements if their speed instructions differ (Fig. $7 A$ ). Comparable results were observed in the subject who performed different amplitude movements while the speed instruction was randomly varied. For example, some of the fast movements to the $5^{\circ}$ target had the same peak velocity (Fig. $8 A$ ), peak acceleration, or peak jerk (Fig. $9 A$ ) as some of the slow movements to the $25^{\circ}$ target. This point is further illustrated by the overlapping of averages of some slow movements to the $25^{\circ}$ target with those of some fast movements to the $5^{\circ}$ target (Fig. 10). Although the 2 sets of movements have the same peak jerk, they have very different durations. These observations indicate that the peak displacement of a step-tracking movement is controlled by adjusting the duration, as well as the magnitude, of the derivatives of displacement.

To further explore this conclusion, we examined the correlations between peak displacement and the product of 2 kinematic variables (derivative magnitude and duration). For movements at all intended speeds to different targets, the correlations between peak displacement and the product of peak velocity and movement duration $(r=0.97-0.99, n=4$; see Fig. $8 B$ ) were striking and were significantly higher than the correlations between peak displacement and peak velocity $(r=0.80-0.95$; see Fig. $8 A$ ). The linear-regression lines in the graphs of peak dis- 


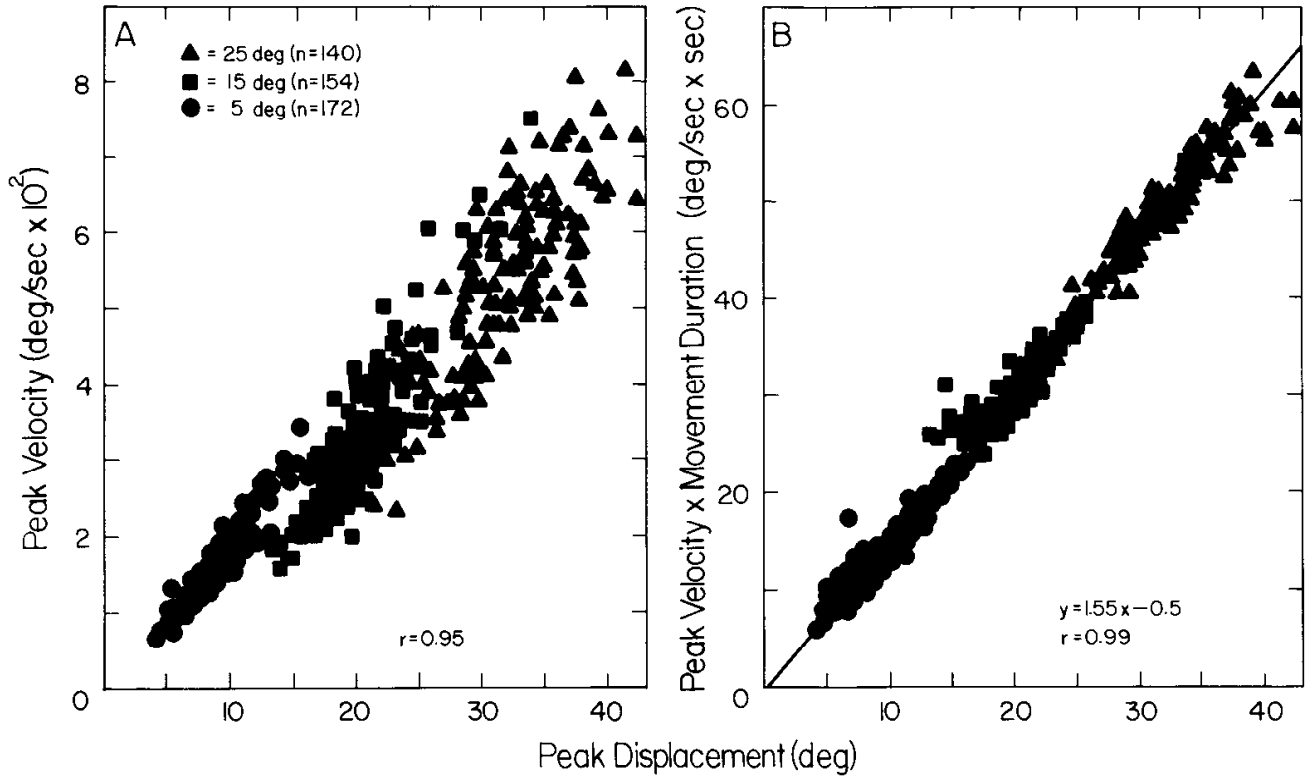

Figure 8. A, Relationship between peak displacement and peak velocity for movements to different targets when intended speed was randomly varied. See Materials and Methods for details of how the speed instruction was varied. $B$, Relationship between peak displacement and the product of peak velocity and movement duration for the same trials as in $A$. placement versus the product of peak velocity and movement duration had an average slope of 1.57. This slope is similar to that calculated for several models of limb movement (Hogan, 1984; Nelson, 1983).

We also examined the results of multiplying peak jerk by movement duration. The correlations between peak displacement and the product of peak jerk and movement duration ( $r=$ $0.67-0.95$; Fig. $9 B$ ) were significantly higher than the correlations between peak displacement and peak jerk $(r=0.38-0.87$; Fig. $9 A$ ). Based on our observations, we hypothesize that, when intended speed varies, peak displacement is controlled by adjusting 2 kinematic variables: (1) the magnitude of the initial positive component of either velocity, acceleration, or jerk; and (2) the duration of this component.

\section{Discussion}

Several major observations were made in this study. First, steptracking movements were performed at a range of intended speeds and were not limited to movements made "as fast as possible." Regardless of the intended speed, the initial trajectory of these movements had a smooth, unimodal velocity pattern that is thought to be characteristic of efficient, skilled movements (see, for example, Nelson, 1983). Second, alterations in the amplitude of step-tracking movements were associated with changes in the magnitudes of the derivatives of displacement (velocity, acceleration, and jerk). Third, alterations in intended speed were associated with changes in derivative duration and derivative magnitude. On the basis of these results, we propose that the initial trajectory of step-tracking movements is generated by central processes that specify both the magnitude and duration of a derivative of displacement. In the following discussion, we will review the evidence for this hypothesis from our own and prior studies.

\section{Control of derivative magnitude}

A number of prior studies have observed that limb velocity is related to movement amplitude (e.g., Cooke, 1980; Freund and Budingen, 1978; Taylor and Birmingham, 1948; Wadman et al., 1979). In a study of rapid finger movements, Freund and Budingen proposed that different amplitude movements are

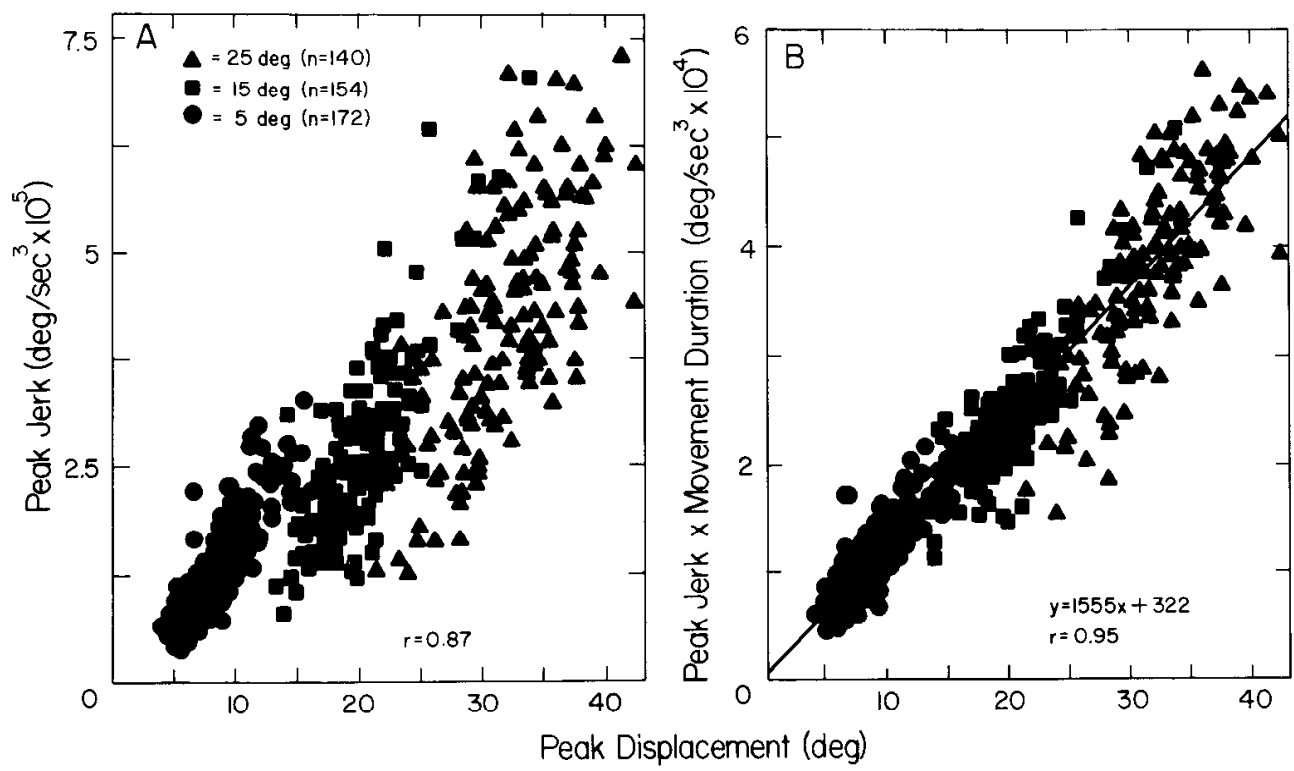

Figure 9. A, Relationship between peak displacement and peak jerk for movements to different targets when intended speed was randomly varied. The same trials were illustrated in Figure 8. $B$, Relationship between peak displacement and the product of peak jerk and movement duration for the same trials as in $A$. 

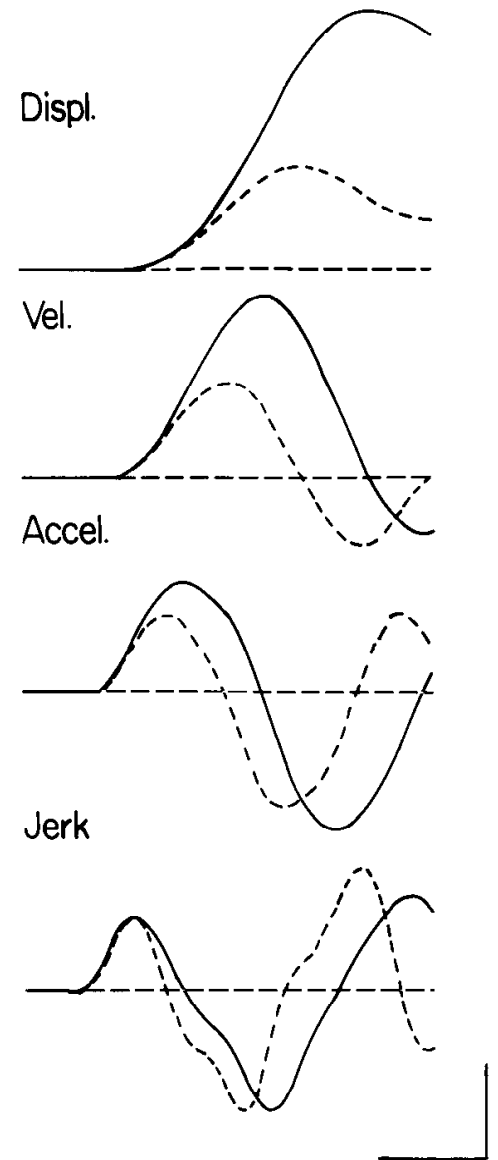

Figure 10. Averages of trials with similar values of peak jerk. Trials were selected from those shown in Figure 9. Solid lines, averages of 21 movements to the $25^{\circ}$ target; dashed lines, averages of 21 movements to the $5^{\circ}$ target. Note that the movements of different amplitude and intended speed can have the same initial values of peak jerk. Displacement, $10^{\circ}$; Velocity, $210 \mathrm{deg} \mathrm{sec}^{-1}$; Acceleration, $7100 \mathrm{deg} \cdot \mathrm{sec}^{-2} ;$ Jerk, $350,000 \mathrm{deg} \cdot \mathrm{sec}^{-3}$. Time, $50 \mathrm{msec}$.

generated by adjusting the velocity, but not the duration, of a limb movement. This has been termed the "speed control" hypothesis (Freund and Budingen, 1978). In the present study, we have observed that peak displacement was highly correlated not only with peak velocity, but also with the peaks of acceleration and jerk (see also Taylor and Birmingham, 1948). In addition, when the speed instruction was varied, we observed changes in the duration, as well as in the magnitude, of the derivatives of displacement. Thus, if the Freund and Budingen hypothesis is to be applied to step-tracking movements, it should be expanded in 2 areas. First, hypotheses about the control of step-tracking movements should not be limited to velocity control. Second, hypotheses about the control of step-tracking movements should include the potential for adjustments in derivative duration, as well as in derivative magnitude.

Because velocity, acceleration, and jerk are graded and phaseadvanced with respect to displacement, feedback related to the derivatives could provide a predictive measure of the initial trajectory's amplitude. Jerk, in particular, reaches its peak very early in a step-tracking movement. Thus, feedback related to the magnitude of jerk could lead to alterations in muscle activity during a movement's later phases. There is evidence that peripheral and central neurons receive signals related to the derivatives of displacement. For example, muscle spindle primarics are particularly sensitive to changes in muscle length (for a review, see Matthews, 1981), and in some circumstances their response appears to signal jerk (Goodwin et al., 1975). Neurons in the sensorimotor cortex also exhibit pronounced sensitivity to the dynamic aspects of imposed movements (for a discussion, see Bedingham and Tatton, 1985). Such peripheral and central signals could be used to monitor and/or adjust step-tracking movements.

Although our observations indicated that the derivatives of displacement are important for generating and monitoring steptracking movements, we were not able to determine which derivative is most significant. We observed that the correlations between the higher derivatives and peak displacement were lower than the correlations between peak velocity and peak displacement. However, we could not measure the higher derivatives of displacement as accurately as we could measure velocity. This is because digital differentiation preferentially amplifies high-frequency noise inherent even in apparently smooth displacement curves (Pezzack et al., 1977; see also Wood, 1982). With each successive differentiation, noise becomes more difficult to separate from the relevant movement signal. Thus, in future experiments, it may be useful to measure the derivatives of displacement more directly.

In companion experiments we observed that peak jerk, unlike the peaks of velocity and acceleration, was uninfluenced by antagonist muscle activity during a step-tracking movement (D. S. Hoffman and P. L. Strick, unpublished observations). Thus, jerk may be a particularly important variable for the nervous system since, during step-tracking movements, it reflects the activity of agonist muscles in isolation from the activity of antagonists. It may also be important to monitor jerk in order to generate the smoothest possible trajectory (Flash and Hogan, 1982; Hogan, 1984).

\section{Control of derivative duration}

Some prior studies have reported that movement duration was nearly constant for rapid movements of different amplitudes (e.g., Freund and Budingen, 1978), whereas other studies have demonstrated changes in this variable with movement amplitude (e.g., Flowers, 1976; Searle and Taylor, 1948; Wadman et al., 1979). When we gave a constant speed instruction, we observed that some subjects performed large-amplitude movements in the same amount of time used for small-amplitude movements. In other subjects, there were changes in movement duration, particularly for "half natural" speed movements. However, these changes were both small and poorly correlated to movement amplitude. Thus, we concur with Searle and Taylor's (1948; pp. 623-624) conclusion about rapid movements, that "the evident tendency is for time of response to remain constant, regardless of distance. ... The slight but reliable increase in duration may be taken as indicating the degree to which complete constancy is not achieved." In addition, we suggest that these statements also apply to movements performed at other intended speeds.

An important observation of the present study is that a change in the speed instruction resulted in a dramatic alteration in the time course of step-tracking movements. For example, changing the speed instruction from move "as fast as possible" to move "at half natural speed" nearly doubled the movement duration in all of our subjects. These results suggest that when human subjects volitionally alter intended speed, they select a different movement duration and, as a result, modify the time course of their movement. These observations also imply that, when animals perform movements to a single target, using a wide range of movement durations (e.g., Flament et al., 1984), these animals are varying their intended speed.

Our results illustrate an important difference between limb and eye movement systems. As noted above, subjects can volitionally alter the time course of step-tracking movements to perform the same wrist displacement. In contrast, subjects lack 
volitional control over the time course of a saccade (Fuchs, 1967; Robinson, 1964, 1981). This difference is not surprising, given the disparate behavioral requirements of eye and limb movements. Howcver, it suggests that there may be some central control mechanisms that are unique to limb movements.

We suggest that movement duration is an important variable which the nervous system controls when it generates and monitors a step-tracking movement. In making this statement, we do not intend to exclude the possibility that other time variables are controlled or monitored by the nervous system. For example, the durations of acceleration and jerk appeared to vary in the same manner as movement duration, although these other durations were not quantitatively examined. Thus, further experiments will be necessary to determine whether the duration of one derivative is more important than another for movement control.

\section{Two-variable control of step-tracking movements}

It should be clear from our results that the initial trajectory of a step-tracking movement cannot be specified by controlling only the magnitude of a single kinematic variable, such as velocity. On the other hand, our data suggest that the specification of peak displacement might be accomplished using the product of 2 kinematic variables. In fact, when we plotted the product of peak velocity and movement duration versus peak displacement, the same regression line fit step-tracking movements that had widely different intended speeds and amplitudes. This suggests that step-tracking movements of different time course and amplitude are generated by the same "control strategy." We propose that the control strategy involves modulating both the magnitude and duration of a derivative of displacement.

The average slope of the regression lines in the graphs of peak displacement versus the product of peak velocity and movement duration was 1.57. This value is similar to that predicted for a linear spring model of joint movement and for models of movement in which either jerk or energy was minimized (Hogan, 1984; Nelson, 1983). Thus, our observations are not inconsistent with the concept that part of the strategy for generating step-tracking movements may involve "optimizing" a physical variable, such as energy or jerk. However, at present there is not enough information to determine which of the models mentioned above would provide the best insight into the central control of step-tracking movements.

Two recent hypotheses about the control of rapid limb movements (Freund and Budingen, 1978; Ghez and Vicario, 1978) have proposed that the magnitude of the initial dynamic phase of movements is graded by modulating the amplitude of an initial "control pulse" (i.e., pulse-height modulation). In contrast, the dynamic phase of saccadic eye movements is thought to be governed by modulating the duration of an initial control pulse (i.e., pulse-width modulation; Robinson, 1981). By comparison, we propose that the nervous system specifies the initial trajectory of a step-tracking movement by controlling both the magnitude and duration of a derivative of displacement. Derivative magnitude is adjusted independently of derivative duration to vary movement amplitude, whereas there is a coordinated adjustment of both variables to vary intended specd. Thus, our hypothesis employs components of both models and, therefore, should be viewed as a form of "pulse-height" and "pulse-width" modulation.

Additional support for our hypothesis comes from companion experiments in which we examined the patterns of muscle activity associated with step-tracking movements of different amplitudes and intended speeds. These movements are produced by well-defined, alternating bursts of activity in agonist and antagonist musles (e.g., Wachholder and Altenburger, 1926). We examined the relations between the magnitudes of the initial agonist and antagonist bursts and various kinematic parameters.
The magnitude of the agonist burst had high correlations with the peaks of velocity, acceleration, and jerk (Hoffman and Strick, 1984). The magnitude of the antagonist burst had a high corrclation with the reciprocal of movement duration (Hoffman and Strick, 1982). These observations suggest that, for the wrist movements we examined, the nervous system generates 2 bursts of muscle activity that provide for control of derivative magnitude (pulse height) and derivative duration (pulse width). These findings will be fully described and discussed in a subsequent publication (D. S. Hoffman and P. L. Strick, unpublished observations).

Our hypothesis will be tested in future experiments that will examine the effects of changes in mechanical conditions on the kinematics of step-tracking movements. We predict that the nervous system will attempt to adjust muscle activity and force (within the performance limits of the system) in order to keep derivative magnitude and duration constant for the same movement made under different mechanical conditions. The present experiments provide some limited support for this prediction. We observed that the slopes of the linear-regression lines relating peak displacement to peak velocity were similar for movements that were opposed (radial deviations) or assisted (ulnar deviations) by gravity. The time course of these movements was also similar. It should be noted, however, that the changes in mechanical conditions caused by gravity were relatively small in our experiments. Other studies have perturbed rapid movements by adding inertial, frictional, or spring-like loads (Bouisset and Lestienne, 1974; Flament et al., 1984; Ghez, 1979). The illustrations of the results of these studies suggest that the nervous system adjusts motor output so that the movements performed under different mechanical conditions have similar kinematics. However, this issue has not been quantitatively analyzed and remains to be more thoroughly examined.

At this point, we can only speculate about the central structures that might be responsible for the control of derivative magnitude and duration. Potentially, many regions of the neuraxis may participate in the generation of step-tracking movements. However, the limited evidence available from studies of patients with motor systems disorders suggests the involvement of the cerebellum and the basal ganglia. For example, patients with cerebellar lesions have dysmetric step-tracking movements (Hallett et al., 1975; see also Brooks and Thach, 1981; Dow and Moruzzi, 1958; Ito, 1984). This symptom could result from an inappropriate adjustment of derivative magnitude and/or duration. A conspicuous symptom of Parkinson's disease is bradykinesia. It has been suggested that this symptom occurs because these patients fail to grade derivative magnitude for different amplitudes of step-tracking movements (Flowers, 1976; Hallett and Khoshbin, 1980). It is our hope that neuron recordings in primates during the performance of step-tracking movements will provide further insights into the central structures involved in the generation and control of step-tracking movements.

\section{References}

Angel, R. W. (1974) Electromyography during voluntary movement: The two-burst pattern. Electroencephalogr. Clin. Neurophysiol. 36: 493-498.

Bedingham, W., and W. G. Tatton (1985) Kinematic representation of imposed forearm movements by pericruciate neurons (areas 4 and $3 a)$ in the awake cat. J. Neurophysiol. 53: 886-909.

Bizzi, E., N. Accornero, W. Chapple, and N. Hogan (1984) Posture control and trajectory formation during arm movement. J. Neurosci. 4: 2738-2744.

Bouisset, S., and F. Lestienne (1974) The organization of a simple voluntary movement as analyzed from its kinematic properties. Brain Res. 71: 451-457.

Brooks, V. B., and W. T. Thach (1981) Cerebellar control of posture and movement. In Handbook of Physiology, sect. 1: The Nervous 
System. Vol. II: Motor Control, V. B. Brooks, ed., pp. 877-946, American Physiological Society, Bethesda, MD.

Brown, S. H. C., and J. D. Cooke (1981) Amplitude- and instructiondependent modulation of movement-related electromyogram activity in humans. J. Physiol. (Lond.) 316: 97-107.

Cooke, J. D. (1980) The organization of simple, skilled movements. In Tutorials in Motor Behavior, G. E. Stclmach and J. Rcquin, cds., pp. 199-212, North-Holland, Amsterdam.

Dow, R. S., and G. Moruzzi (1958) The Physiology and Pathology of the Cerebellum. U. Minnesota Press, Minneapolis, MN.

Flament, D., J. Hore, and T. Vilis (1984) Braking of fast and accurate elbow flexions in the monkey. J. Physiol. (Lond.) 349: 195-203.

Flash, T., and N. Hogan (1982) Evidence for an optimization strategy in arm trajectory formation. Soc. Neurosci. Abstr. 8: 282.

Flowers, K. S. (1976) Visual "closed-loop" and "open-loop" characteristics of voluntary movements in patients with Parkinson's discase and intention tremor. Brain 99: 269-310.

Freund, H.-J., and H. J. Budingen (1978) The relationship between speed and amplitude of the fastest voluntary contractions of human arm muscles. Exp. Brain Res. 31: 1-12.

Fuchs, A. F. (1967) Saccadic and smooth pursuit eye movements in the monkey. J. Physiol. (Lond.) 191: 609-631.

Ghez, C. (1979) Contributions of central programs to rapid movement in the cat. In Integration in the Nervous System, $\mathrm{H}$. Asanuma and V. J. Wilson, eds., pp. 305-319, Igaku-Shoin, Tokyo.

Ghez, C., and D. Vicario (1978) The control of rapid limb movement in the cat. II. Scaling of isometric force adjustments. Exp. Brain Res. 33: 191-202.

Goodwin, G. M., M. Hullinger, and P. B. C. Matthews (1975) The effects of fusimotor stimulation during small amplitude stretching on the frequency-response of the primary ending of the mammalian muscle spindle. J. Physiol. (Lond.) 253: 175-206.

Hallett, M., and S. Khoshbin (1980) A physiological mechanism of bradykinesia. Brain 103: 301-314.

Hallett, M., and C. D. Marsden (1979) Ballistic flexion movements of the human thumb. J. Physiol. (Lond.) 294: 33-50.

Hallett, M., B. T. Shahani, and R. R. Young (1975) EMG analysis of stereotyped voluntary movements in man. J. Neurol. Neurosurg. Psychiatry 38 : 1154-1162.

Hoffman, D. S., and P. L. Strick (1982) Evidence that antagonist muscle activity is centrally generated. Soc. Neurosci. Abstr. 8: 733 .

Hoffman, D. S., and P. L. Strick (1984) Controlled variables in steptracking movements of the wrist. Soc. Neurosci. Abstr. 10: 332.

Hogan, N. (1984) An organizing principle for a class of voluntary movements. J. Neurosci. 4: 2745-2754.

Ito, M. (1984) The Cerehellum and Neural Control, Raven, New York.

Lestienne, F. (1979) Effects of inertial load and velocity on the braking process of voluntary limb movements. Exp. Brain Res. 35: 407-418.
Lestienne, F., A. Polit, and E. Bizzi (1981) Functional organization of the motor process underlying the transition from movement to posture. Brain Res. 230: 121-131.

Marsden, C. D., J. A. Obeso, and J. C. Rothwell (1983) The function of the antagonist muscle during fast limb movements in man. J. Physiol. (Lond.) 335: 1-13.

Matthews, P. B. C. (1981) Muscle spindles: Their messages and their fusimotor supply. In Handbook of Physiology, sect. 1: The Nervous System, Vol. II: Motor Control, V. B. Brooks, ed., pp. 189-228, American Physiological Society, Bethesda, MD.

Nelson, W. L. (1983) Physical principles for economies of skilled movements. Biol. Cybern. 46: 135-147.

Pezzack, J. C., R. W. Norman, and D. A. Winter (1977) An assessment of derivative determining techniques used for motion analysis. J. Biomech. 10: 377-382.

Polit, A., and E. Bizzi (1979) Characteristics of the motor programs underlying arm movements in monkeys. J. Neurophysiol. 42: 183194.

Robinson, D. A. (1964) The mechanics of human saccadic eye movement. J. Physiol. (Lond.) 174: 245-264.

Robinson, D. $\Lambda$. (1981) Control of eye movements. In Handbook of Physiology, sect. 1: The Nervous System, Vol. II: Motor Control, V. B. Brooks, ed., pp. 1275-1320, American Physiological Society, Bethesda, MD.

Searle, L. V., and F. V. Taylor (1948) Studies of tracking behavior. I. Rate and time characteristics of simple corrective movements. J. Exp. Psychol. 38: 615-631.

Stein, R. B. (1982) What muscle variable(s) does the nervous system control in limb movements? Behav. Brain Sci. 5: 535-577.

Taylor, F. V., and H. P. Birmingham (1948) Studies of tracking behavior. II. The acceleration pattern of quick manual corrective responses. J. Exp. Psychol. 38: 783-795.

Wachholder, K. (1928) Willkürliche Haltung und Bewegung, insbesondere im Lichte elektrophysiologischer Untersuchungen. Ergeb. Physiol. 26: 568-775.

Wachholder, K., and H. Altenburger (1926) Beiträge zur Physiologie der willkürlichen Bewegung. X. Einzelbewegungen. Pflugers Arch. 214: 642-661.

Wadman, W. J., J. J. Denier van der Gon, R. H. Geuze, and C. R. Mol (1979) Control of fast goal-directed arm movements. J. Hum. Move. Stud. 5: 3-17.

Waters, P., and P. L. Strick (1981) Influence of "strategy" on muscle activity during ballistic movements. Brain Res. 207: 189-194.

Wood, G. A. (1982) Data smoothing and differentiation procedures in biomechanics. Exerc. Sport Sci. Rev. 10: 308-362. 